\title{
LES ETHMOIDITES AIGUES EXTERIORISEES DE L'ENFANT ETUDE DE 11 OBSERVATIONS
}

\section{ACUTE ETHMOIDITIS IN CHILDREN ABOUT 11 CASES}

\author{
L. BOUGHAMOURA, F. HMILA, M. BEN ALI*, I. CHABCHOUB, J. BOUGUILA, \\ M. YACOUB, K. BOUZOUITA*, A S. ESSOUSSI. \\ SERVICE DE PÉDIATRIE CHU F. HACHED SOUSSE \\ *SERVICE ORL CHU F. HACHED SOUSSE
}

\begin{abstract}
RESUME
Les ethmoïdites sont les sinusites les plus fréquentes chez l'enfant de moins de 3 ans.

La gravité de cette affection est liée à l'extériorisation orbito-oculaire et endocrânienne de l'infection.

But : Analyser les caractéristiques clinico- radiologiques et thérapeutiques de cette affection.

Patients et méthodes : Il s'agit d'une étude rétrospective portant sur 11 enfants hospitalisés pour ethmoïdite aigue, dans le service de pédiatrie du CHU Farhat Hached de Sousse, durant une période de 6 ans.

Résultats : L'âge moyen de nos patients était de 4 ans et 1/2, ils se répartissaient en 8 filles et 3 garçons. Le délai de consultation était de trois jours en moyenne. La fièvre et l'œdème palpébral étaient deux critères constants chez tous nos patients.

L'atteinte était unilatérale dans neuf cas, et bilatérale dans deux cas. La TDM éthmoïdo-orbitaire et cérébrale, réalisée pour tous les patients, nous a permis de confirmer le diagnostic et de préciser l'extension locorégionale. Une cellulite préseptale, une cellulite orbitaire, un abcès sous périosté étaient retrouvées, dans trois cas et une collection orbitaire dans les deux autres. Une bithérapie (céfotaxime-fosfomycine) était prescrite de première intention chez 10 patients. La metronidazole a été associée dans 3 cas Un drainage chirurgical était réalisé chez quatre patients. L'évolution était favorable dans tous les cas.

Conclusion : contenu de la gravité et les germes rencontrés dans cette affection, l'antibiothérapie de première intention doit être large, précoce et intensive. Le recours au drainage est impératif en cas de collection orbitaire.
\end{abstract}

\section{SUMMARY}

Acute ethmoiditis are bacterial infections of ethmoid sinuses, which may spread to the orbital or the endocranial spaces. They are the most frequent among bacterial sinusitis before 3 years.

Population and methods:Our study is retrospective, it included all children hospitalized in our pediatric unit from 2000 to 2006 for an acute ethmoiditis. We analyzed clinical and radiological presentations, and discussed therapeutic modalities used to treat this pathology.

Results:Over this 6-years period, 11 children (mean age 4.5 years) where hospitalized for acute ethmoïdites. They were 3 boy and 8 girls. All patients presented with high fever and palpabral oedems. Etmoïditis was unilateral in 9 Children and bilateral in two. Ethmoïdio-orbital CT scan confirmed acute ethmoïdis, the cerebral one assessed intra-cranial diffusion. The acute ethmoidis was complicated with, preseptal cellulites in three cases, orbital cellulitis in three cases, subperiostal abscess in three others and orbital collection in two patients. Most children received an association of cefotaxisme and fosfomycemie for a mean duration of 13 days. Metronidazole was associated to the previous antibiotherapy in three cases. Four patients, required surgical treatment in addition to antibiotherapy.All children saw favorable out come.

Conclusion:It is always difficult to found the bacteria responsible for an acute ethmoiditis. The first antibiotic therapy must be chosen to be effective in the most frequently responsible bacteria tacking in consideration the potentiel severity of this sinusitis.

\section{INTRODUCTION}

L'ethmoïdite aiguë (EA) est l'infection bactérienne des cellules du labyrinthe ethmoïdal. II s'agit d'une infection sévère, nécessitant un diagnostic et un traitement urgents du fait du risque de complications ophtalmologiques et endocrâniennes [1]. Elle survient le plus souvent entre l'âge de 6 mois et 5 ans. Le signe d'appel est l'apparition dans un contexte fébrile d'un œdème palpébral [2]. Le traitement repose essentiellement sur l'antibiothérapie à large spectre. La chirurgie est réservée aux formes compliquées. Le but de notre étude est d'analyser les caractéristiques clinico-radiologiques et thérapeutiques de cette affection à travers 11 observations et une revue de la littérature. 


\section{PATIENTS ET METHODES}

Nous avons mené une étude rétrospective à propos de 11 cas d'ethmoïdite aiguë colligés sur une période de 6 ans (Mars 2000 - Mars 2006) dans le service de pédiatrie du CHU Farhat Hached de Sousse. Le diagnostic était porté sur la présence d'un œdème palpébral siégeant ou prédominant au niveau de la paupière supérieure avec des signes inflammatoires locaux, le tout évoluant dans un contexte fébrile.

Une TDM éthmoido-orbitaire et cérébrale était réalisée pour tous les enfants.

\section{RESULTATS}

L'âge moyen de nos patients était de 4 ans et demi avec des extrêmes allant de 2 mois à 13 ans. La majorité était d'âge inférieur ou égal à 3 ans ( 7 cas/11).

II s'agissait de 8 filles et 3 garçons. Concernant les antécédents, une patiente était traitée pour leucémie aigue lymphoblastique avant cinq ans et était en rémission complète. Un autre patient avait un rétrécissement pulmonaire congénital asymptomatique. Une prise préalable d'antibiotique était retrouvée chez 6 patients.

Le motif de consultation était un œdème palpébral chez tous les patients. Le délai moyen de consultation était de 3 jours avec des extrêmes allant de 1 à 8 jours.

La fièvre était présente chez tous les patients avant l'hospitalisation, ou retrouvée à l'examen physique. Deux enfants se plaignaient de céphalées, âgés respectivement de 10 et 13 ans,dont une avait une diplopie. A l'admission, tous les enfants avaient un œdème de la paupière supérieure. L'œdème prédominait au niveau de la paupière supérieure et de l'angle interne de l'œil dans six cas (Fig. 1), alors qu'il intéressait les deux paupières dans cinq cas. II était unilatéral chez neuf patients, et bilatéral chez les deux autres.

Une exophtalmie unilatérale et un chémosis étaient présents chez cinq patients, une baisse de l'acuité visuelle chez une patiente.

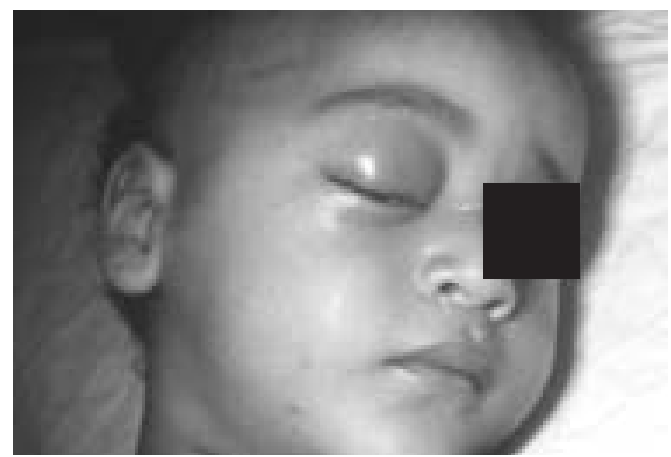

Fig. 1 : Oedème inflammatoire de la paupière supérieure prédominant à l'angle interne de l'œil.

L'examen ORL avait objectivé une rhinopharyngite dans cinq cas. L'endoscopie nasale n'a pu être réalisée que chez trois patients objectivant du pus au niveau du méat moyen.

Devant ce contexte clinique le diagnostic d'ethmoïdite aiguë extériorisée était fortement suspecté motivant la demande d'une TDM éthmoido-orbitaire et cérébrale, réalisée en urgence chez tous nos patients. Cet examen avait permis de confirmer le diagnostic d'ethmoïdite aigue dans tous les cas en objectivant un comblement des cellules ethmoïdales unilatéral dans neuf cas et bilatéral dans deux cas. (Fig 2)

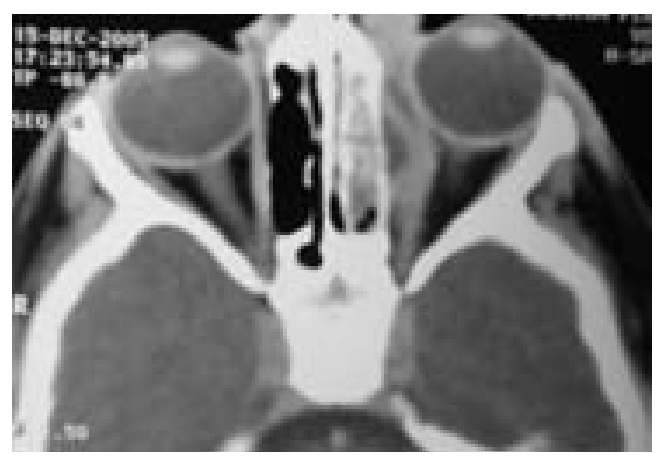

Fig. 2 : TDM éthmoido orbitaire, coupe axiale : comblement des cellules éthmoidales, abcés sous périosté.

Elle nous a permis de classer nos patients selon la classification de CHANDLER en : cellulite pré septale, cellulite orbitaire, abcès sous périosté dans respectivement trois cas et abcès orbitaire dans deux cas. Cet examen avait aussi mis en évidence l'atteinte associée des sinus maxillaires (6 cas), sinus sphénoïdaux (2 cas) et d'une pansinusite chez un patient.

Sur le plan biologique, la $C$ réactive protéine (CRP) était en moyenne de $57 \mathrm{mg} / \mathrm{ml}$, une hyperleucocytose à prédominance polynucléaires neutrophiles était retrouvée chez 8 patients. L'hémoculture réalisée chez 6 patients était constamment négative.

Le prélèvement au niveau des fosses nasales n'était réalisé que chez deux patients, il était négatif.

Dès l'admission dix patients avaient reçu une biantibiothérapie par voie intra veineuse associant céfotaxime $(100-200 \mathrm{mg} / \mathrm{kg} / \mathrm{j})$ et fosfomycine $(100-200 \mathrm{mg} / \mathrm{kg} / \mathrm{j})$. Le métronidazole (Flagyl $\left.{ }^{\circledR}\right)$ était associé chez trois patients. Un seul patient avait reçu de première intention l'association céfotaxime, vancomycine et métronidazole vue la gravité du tableau clinique initial et par crainte d'un pneumocoque de sensibilité diminuée. La durée moyenne de cette antibiothérapie était de 13 jours avec des extrêmes allant de 8 à 23 jours. Un relais par voie orale, par l'association amoxicilline - acide clavulanique, était prescrit chez 8 patients et par l'oxacilline chez un patient.

Nous avons eu recours à un drainage chirurgical chez quatre patients dans les premières 48 heures. II a consisté en une orbitotomie avec décollement sous périosté et évacuation de la collection.

L'évolution était favorable chez tous nos patients. 


\section{DISCUSSION}

Les cavités sinusiennes se forment progressivement pendant la petite enfance et ce sont les cellules ethmoïdales qui sont les premières à apparaître dès les premiers mois de vie. De ce fait, les ethmoïdites sont les sinusites les plus fréquentes chez l'enfant de moins de 3 ans [3]. En effet, plusieurs éléments anatomiques et physiopathologiques favorisent cette pathologie dans cette tranche d'âge.

C'est une infection sévère, nécessitant un diagnostic et un traitement urgents du fait du risque de complications ophtalmologiques et endocrâniennes [4].

L'infection peut se propager à l'orbite soit par contact direct au travers de la lame papyracée extrêmement fine chez l'enfant, soit par l'intermédiaire du réseau veineux périorbitaire.

L'altération de l'acuité visuelle, voire la cécité peut s'expliquer essentiellement par deux phénomènes : une névrite orbitaire secondaire à l'inflammation du globe et une occlusion de la veine ophtalmique supérieure. Cette extension orbitaire a été classée par CHANDLER [4] en cinq stades (tableau I). II s'agit d'une classification anatomoclinique qui distingue deux entités différentes : les cellulites périorbitaires ou préseptales (stade1), situées en avant du septum orbitaire, d'évolution le plus souvent favorable sous traitement médical, et les cellulites rétroseptales (stades 2 à 5), plus rares, plus graves pouvant mettre en jeu le pronostic vital et fonctionnel [5].

\begin{tabular}{|c|c|l|}
\hline Classification & Localisation & \multicolumn{1}{|c|}{ Clinique } \\
\hline Stade 1 & Cellulite préseptale & Oedème palpébral isolé \\
\hline Stade 2 & Cellulite orbitaire & \multicolumn{1}{c|}{ Chémosis, exophtalmie } \\
\hline Stade 3 & Abcès sous périosté & $\begin{array}{l}\text { Baisse de l'acuité visuelle } \\
\text { Diminution des mouvements } \\
\text { oculaires }\end{array}$ \\
\hline Stade 4 & Abcès orbitaire & $\begin{array}{l}\text { Baisse importante de l'acuité } \\
\text { visuelle } \\
\text { Cécité ; atteinte oculaire }\end{array}$ \\
\hline Stade 5 & Thrombose du sinus & $\begin{array}{l}\text { controlatérale ; syndrome } \\
\text { méningé }\end{array}$ \\
\hline
\end{tabular}

Tableau I : Classification de Chandler [4]

L'ethmoïdite aiguë survient le plus fréquemment chez l'enfant de 2 à 3 ans, mais elle peut se voir plus précocement à l'âge de 4 mois [1] et aussi chez l'enfant plus âgé [6].

Dans notre série, sept patients avaient un âge inférieur ou égal à 3 ans, dont le plus jeune âgé de 2 mois. Nous avons noté une prédominance féminine $(8 / 3)$, ceci a été rapportée par certains auteurs [2, 6].

L'œdème palpébral est le signe le plus constant, précédé ou accompagné dans la plupart des cas d'une infection rhinopharyngée, mais l'absence de rhinorrhée ne doit pas exclure le diagnostic. Cet œdème palpébral souvent uni- latéral, avec des signes inflammatoires locaux prédominant à la paupière supérieure et à l'angle interne de l'œil [8]. II s'étend plus ou moins vite à la paupière inférieure puis aux paupières controlatérales [2]. L'hyperthermie n'est pas constante, elle peut manquer en raison du traitement antipyrétique et antibiotique préalablement administré [7]. Une obstruction nasale et un mouchage purulent sont inconstants.

Pour nos malades, cet œdème était constant, prédominant au niveau de la paupière supérieure chez six patients.

La fréquence des signes oculaires : conjonctivite, chémosis, exophtalmie explique que l'ophtalmologiste soit fréquemment consulté en première intention. Les céphalées ne sont notées que dans $20 \%$ des cas du fait que dans la majorité des cas, les enfants sont âgés de moins de 3 ans et ne peuvent pas manifester ce symptôme [7]. Les céphalées étaient rapportées par deux de nos patients âgés respectivement de 10 et 13 ans.

L'examen ophtalmologique paraît indispensable dans le bilan de tout œdème périorbitaire, il va permettre d'évaluer l'acuité visuelle et la surveiller, sa baisse est en rapport avec une atteinte rétroseptale. Elle pourrait en cas d'altération importante ( inf . à 3/10) poser, à elle seule, une indication chirurgicale de drainage [3]. Mais sur le plan pratique, elle ne peut être mesurée que pour les grands enfants.

Les autres signes, bien que tardifs (paralysie oculomotrice, hypoesthésie cornéenne, et mydriase aréflexique) prennent alors toute leur valeur [3].

Sur le plan bactériologique, l'enquête bactériologique est le plus souvent décevante au cours des ethmoïdites aiguës extériorisées. Les seuls prélèvements qui seraient faciles à réaliser, le prélèvement endonasal et le prélèvement nasopharyngé, n'ont aucune valeur [2]. Le prélèvement le plus intéressant est celui des abcès sous périostés ou orbitaires ce qui n'est pas le cas de toutes les ethmoïdites aiguës extériorisées. L'abcès doit être assez volumineux pour pouvoir être ponctionné (dans notre série, l'abcès a été considéré, au vu de l'examen tomodensitométrique, comme impossible à ponctionner dans 1 cas/5).

Les germes les plus fréquemment retrouvés dans les prélèvements par ponction d'abcès sous-périosté sont Streptococcus pneumoniae, Haemophilus influenzae et Staphylococcus auréus [2, 9]. Pour Brook [9], dans sa série qui compte 26 malades, les anaérobies étaient retrouvés dans $22 \%$ des cas. Cependant, François [2], n'en a isolé aucun dans sa série.

La ponction lombaire à la recherche d'une localisation méningée a été recommandée par certains auteurs de façon systématique en présence de signes méningés ou lorsque l'âge de l'enfant est inférieur ou égal à 6 mois [3]. Par ailleurs, les hémocultures restent stériles dans la majorité des cas. Pour François [2], dans une série de 
125 cas d'ethmoïdites aigues extériorisées, l'hémoculture était positive uniquement dans $8,75 \%$ des cas.

La radiographie standard a un intérêt limité avant l'âge de 2 ans, en raison de la difficulté d'interprétation [10]. En fait, tout œdème palpébral dans un contexte d'infection rhinopharyngée chez l'enfant impose un scanner en urgence [1, 7].

La tomodensitométrie permet un diagnostic positif et un bilan lésionnel de certitude, en étudiant l'ensemble des cavités sinusiennes, des éléments intra-orbitaires et des structures intra-craniennes. L'injection de produit de contraste permet de mieux étudier la nature d'une anomalie rétroseptale. Elle permet aussi la recherche de thrombose des veines ophtalmiques et du sinus caverneux $[1,7]$.

Les données tomodensitométriques sont indispensables pour indiquer et guider un éventuel geste chirurgical.

Pour nos patients, la TDM avait confirmé le diagnostic dans tous les cas et avait mis en évidence l'atteinte associée des autres sinus. D'autre part, elle avait montré des signes en faveur d'une cellulite préseptale (3 cas), cellulite périorbitaire ( 3 cas), d'abcès sous périosté ( 3 cas) et d'abcès orbitaire ( 2 cas).

L'antibiothérapie probabiliste par voie parentérale à large spectre associant le céfotaxime et la fosfomycine, paraît l'attitude la plus logique [2]. Cette association couvre pratiquement tous les germes susceptibles d'être responsables d'une ethmoïdite aiguë extériorisée chez l'enfant, en particulier $\mathrm{H}$. influenzae sécréteur de bétalactamase, S. pneumoniae éventuellement de sensibilité diminuée à la pénicilline (PSDP) et $S$. auréus qu'il soit méticilline sensible ou méticilline résistant (SARM) [2].

Cependant, certains auteurs recommandent en première intention l'association amoxicilline - acide clavulanique [11]. Cette antibiothérapie est inefficace sur les PSDP. D'autres [12,13] préconisent une monothérapie par céfotaxime, avec cependant un risque d'inefficacité sur le SARM.

Enfin, la sensibilité inconstante des anaérobies (en particulier Bacteroides fragilis) à l'association amoxicilline- acide clavulanique justifie pour certains auteurs l'association d'un imidazolé. Le metronidazole n'a été associé que chez 4 de nos patients.

La durée du traitement intraveineux est fonction de l'évolution clinique. II faut attendre l'apyrexie et la diminution nette de l'œdème palpébral pour arrêter le traitement parentéral. Elle varie de 3 à 15 jours, en fonction de la clinique $[2,3,14]$. Le relais est pris en suite par voie orale pour une durée de 5 à 15 jours [2]. Le traitement sera d'autant plus long que les signes cliniques ont mis plus longtemps à s'amender sous traitement antibiotique parentéral. Le relais a été pris par l'association amoxicilline - acide clavulanique dans $90 \%$ des cas pour François [2] et dans $64 \%$ des cas dans notre série.

En cas d'allergie aux bêtalactamines, le relais peut être pris par la pristinamycine après l'âge de 6 ans, par le cotrimoxazole au dessous de cet âge.

Le traitement chirurgical est indiqué lors d'abcès sous périosté ou orbitaire, en association avec une antibiothérapie. II consiste en un drainage réalisé par orbitotomie externe seule, avec incision du périoste, ou associée à une ethmoïdectomie endonasale [9].

Le pronostic des ethmoïdites dépend de la précocité du diagnostic et du stade d'évolution anatomo-clinique.

\section{CONCLUSION}

Les ethmoïdites aiguës extériorisées sont des infections bactériennes qui exposent à un risque de diffusion de l'infection à l'orbite et aux espaces endocriniens.

Le traitement est essentiellement médical basé sur une antibiothérapie adaptée aux germes les plus fréquemment rencontrés. Ce traitement doit être débuté rapidement afin d'empêcher l'extension de l'infection et d'éviter l'apparition des complications.

\section{REFERENCES}

[1] Oxford LE, Mc Clay J. Complications of acute sinusitis in children. Otolaryng-Head and Neck Surgery 2005; 133 : 32-7.

[2] François $M$, Mariani-Kurkdjian P, Dupont E, Bingen E. Ethmoïdites aiguës extériorisées de l'enfant : à propos d'une série de 125 cas. Arch. Pédiatr. 2005 ; $13: 6-10$.

[3] Aïdan P, François M, Prunel M, Narcy P. Cellulite de la région orbitaire chez l'enfant. Arch. Pédiatr. $1994 ; 1: 879-85$.

[4] Chandler JR, Langenbrunner DJ, Stevens ER. The pathogenesis of orbital complications in acute sinusitis. Laryngoscope $1970 ; 80: 1414-28$.

[5] Ployet MJ, Borderson Y, Capelier M, Girard M. Cellulite de la face. In: Garabedian JN, éd. Infection ORL de l'enfant, 1ère éd. Paris ; 1990 : 63-72.

[6] Pinzuti-Rodné V, Elmaleh M, François M, Williams M, Narcy P, Hassan M. Intérêt de l'échographie orbitaire dans les ethmoïdites de l'enfant. J. Radiol. 1999 ; $80: 569-74$.

[7] Chobaut JC, Menget A, Badet JM et al. Les ethmoïdites de l'enfant à propos de 20 observations récentes. J. F. ORL 1993; 42 : 195-200.
[8] Sinusite aigue de l'enfant.Méd.Mal.Inf 2001; 31 :508s-21s.

[9] Brook I. Bacteriology of acute and chronic ethmoid sinusitis. J. Clin. Microbiol. 2005; 43: 3479-80.

[10] Schramm VL, Curtin HD, Kennerdell JS. Evaluation of orbital cellulitis and results of treatment. Laryngoscope $1982 ; 92: 732-8$.

[11] Clement PA, Bluestone CD, Gordts F et al. Management of rhinosinusitis in children, consensus meeting, Brussels, Belgium. Arch. Otolaryngol. Head Neck. Surg. $1998 ; 124: 31-4$.

[12] Sobol SE, Marchaud J, Tewfik TL et al. Orbital complications of sinusitis in children. J. Otolaryngol. $2003 ; 31: 131-6$.

[13] Froehlich. Ethomoïdite aiguë de l'enfant : diagnostic et prise en charge des complications. Cahiers ORL $2001 ; 35: 271-2$.

[14] François $M$. Traitement de première intention des ethmoïdites de l'enfant. Cahiers ORL $2001 ; 35: 269-70$.

[15] Peytral C, Chevalier. Complications ophtalmologiques en pathologie oto-rhinolaryngologique. EMC Oto-Rhino-laryngologie. 2004 ; 1 : 199-224. 


\title{
LA GRANULOMATOSE DE WEGENER A PROPOS DE TROIS LOCALISATIONS ORL RARES
}

\section{RARE ENT MANIFESTATION OF WEGENER'S GRANULOMATOSIS}

\author{
S. TRABELSI, S. KHARRAT, S. BEN ALI, S. SAHTOUT, L. OUERTATANI, S. ATALLAH, N. BEN NEJI, \\ N. BELTAIEF, G. BESBES, S. HACHICHA. \\ SERVICE ORL ET CMF - HÔPITAL LA RABTA, TUNIS
}

\begin{abstract}
La granulomatose de Wegener (GW) est une vascularite nécrosante granulomateuse présentant un tropisme particulier pour les voies aériennes supérieures.

Nous rapportons 3 cas de la GW correspondants à des localisations ORL rares de la maladie : orbito-meningée, laryngée et linguale.

Le but de nos études est d'analyser les particularités épidemiologiques et cliniques de cette pathologie.
\end{abstract}

\section{SUMMARY}

Wegener's Granulomatosis (WG) is a necrotizing granulomatous vasculitis that has a strong affinity for the upper respiratory tract. We report 3cases of rare ORL locations of Wegener's Granulomatosis (orbit, larynx and tongue). The aim of our study is to analyse the characteristic features of WG.

\section{INTRODUCTION}

Décrite par Wegener en 1936, la granulomatose de Wegener (GW) est une vascularite nécrosante de cause inconnue, touchant les voies respiratoires et le rein.

Sa prévalence a été estimée à 3 cas pour 100000 personnes (1).

Le tableau clinique de la GW est dominé par l'atteinte $\mathrm{ORL}$ et pulmonaire précoce, révélatrices dans plus de $70 \%$ des cas, et une atteinte rénale plus tardive qui détermine le pronostic (2).

Les manifestations ORL sont pratiquement constantes, touchant préférentiellement les cavités naso-sinusiennes $(2,3,4)$.

Certaines localisations demeurent rares voire exceptionnelles, nous en rapportons trois observations à propos de localisations orbito-méningée, laryngée et linguale.

\section{RESULTATS \\ OBSERVATION 1 :}

Un jeune de 26 ans, a consulté en avril 2003 pour une exophtalmie droite douloureuse associée à une rougeur oculaire et des céphalées en casque.

L'examen clinique trouvait un œil droit rouge avec une exophtalmie droite non réductible et douloureuse. L'endoscopie nasale a révélé une muqueuse pituitaire congestive avec du pus en regard du méat moyen droit. Une imagerie (TDM, IRM) a été pratiquée montrant un processus lésionnel du quadrant supéro-externe de l'orbite droite associé à une infiltration méningée en regard (Fig. 1, 2, 3).

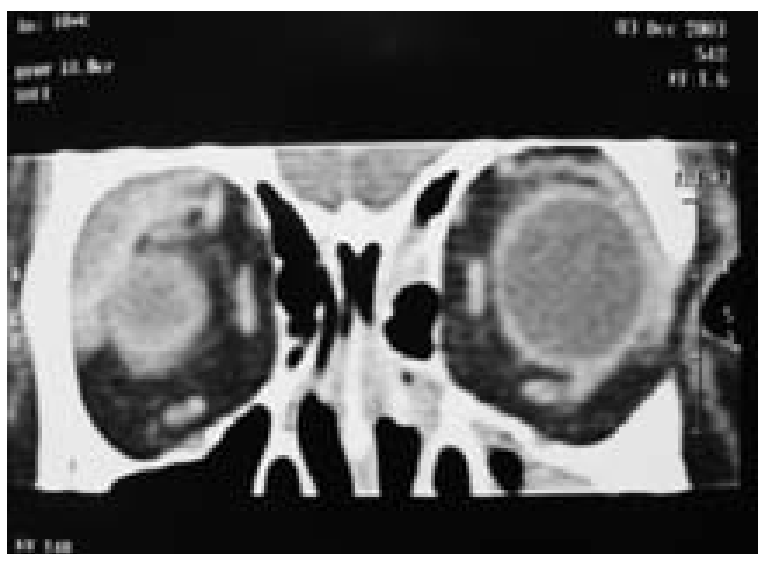

Fig. 1 : TDM du massif facial en coupe coronale : lésion du quadrant supéro-externe de L'orbite droit

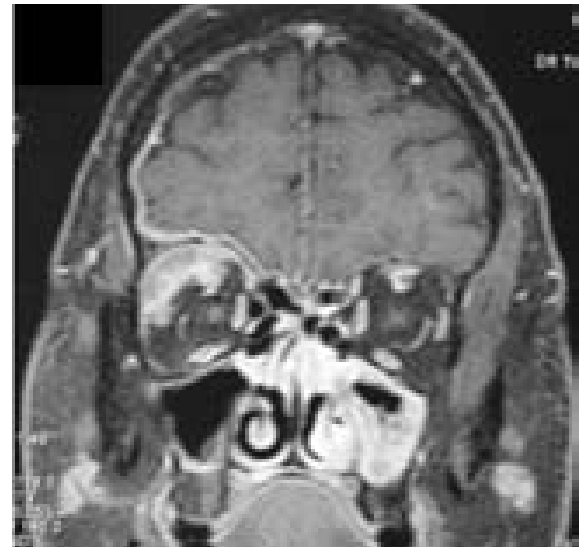

Fig. 2 : IRM en coupe frontale : lésion orbitaire droite en hyposignal 


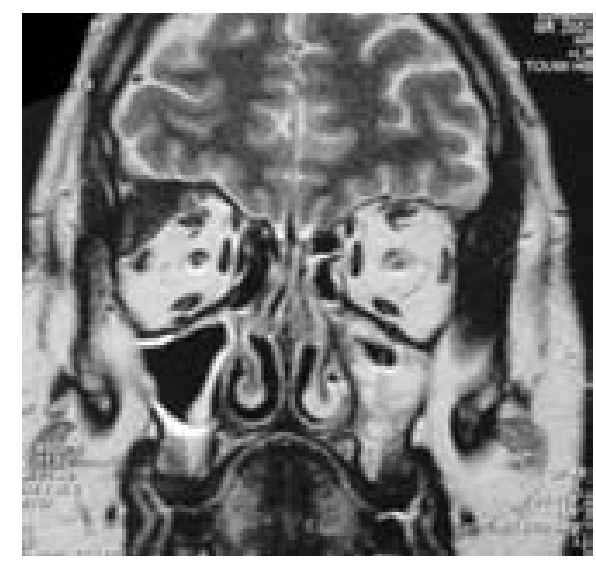

Fig. 3 : IRM du massif facial en coupe coronale : lésion orbitaire droite en hyposignal T2 avec prise de contraste méningée

Une biopsie orbitaire a conclu au diagnostic d'une pseudotumeur inflammatoire.

Un mois plus tard, l'évolution a été marquée par la survenue d'une hématurie, de crachats hémoptoïques et l'apparition de lésions de purpura vasculaire au niveau des membres inférieurs.

La biologie a montré un syndrome inflammatoire avec des c-ANCA positifs de type PR3.

La biopsie des lésions cutanées a conclu à une vascularite leucocytoclasique et la ponction biopsie rénale a mis en évidence un aspect de glomérulonéphrite extra-capsulaire.

Le diagnostic de maladie de Wegener a été retenu.

Le patient a été mis sous cyclophosphomide et corticoïdes.

Après un recul de 24 mois, l'évolution s'est faite vers la stabilisation clinique avec amendement de la symptomatologie respiratoire, disparition du purpura, et normalisation de la fonction rénale.

OBSERVATION 2 :

Un homme de 44 ans, tabagique à 30 PA, sans antécédents pathologiques notables, a consulté en mai 2002 pour une dysphonie intermittente isolée évoluant depuis un an.

L'endoscopie a montré une formation bourgeonnante intéressant la corde vocale gauche, la bande ventriculaire homolatérale et la commissure antérieure avec une mobilité conservée des deux cordes vocales.

Le reste de l'examen ORL était normal, en particulier les aires ganglionnaires étaient libres.

La TDM a objectivé un rétrécissement de la lumière laryngée à l'étage glottique du à une infiltration des bandes ventriculaires et des cordes vocales avec densification de la graisse paralaryngée en regard (Fig. $n^{\circ} 4$ ).

L'étude anatomopathologique de la biopsie laryngée pratiquée, a conclu à une localisation laryngée de la maladie de Wegener. Le bilan d'extension a retrouvé une atteinte renale et pulmonaire. Le patient a été perdu de vue avant traitement.

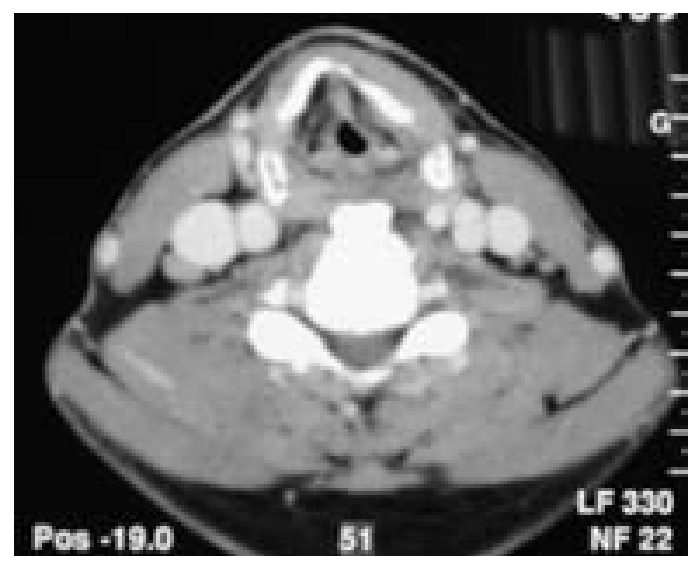

Fig. 4 : TDM laryngé en coupe transversale : rétrécissement de la lumière laryngée à l'étage glottique

\section{OBSERVATION 3 :}

Une femme âgée de 52 ans, sans antécédents notables, a consulté en octobre 2000 pour une lésion de la face dorsale de la langue évoluant depuis 6 mois avec notion d'un amaigrissement modéré non chiffré.

A l'examen, il existait une lésion pseudopolypoide bourgeonnante, non ulcérée, indolore de la face dorsale de la langue mobile en avant du $\mathrm{V}$ lingual (Fig. 5). La mobilité de la langue est conservée. Les aires ganglionnaires cervicales étaient libres.

Le reste de l'examen $O R L$ et somatique était sans particularités.

Une biopsie de la langue a été réalisée évoquant le diagnostic de GW.

Un bilan à la recherche d'autres localisations de la maladie a été pratiqué retrouvant un foyer nasosinusien suspecté à la TDM a été confirmé par la biopsie nasale. L'exploration pulmonaire était négative.Une hématurie microscopique a été trouvée sans protéinurie. Le bilan biologique a objectivé un syndrome inflammatoire. Le dosage des anticorps anticytoplasmes des polynucléaires neutrophiles (ANCA) était positif.

Devant les données cliniques, biologiques et histologiques, le diagnostic de GW a été retenu mais la patiente a été perdue de vue.

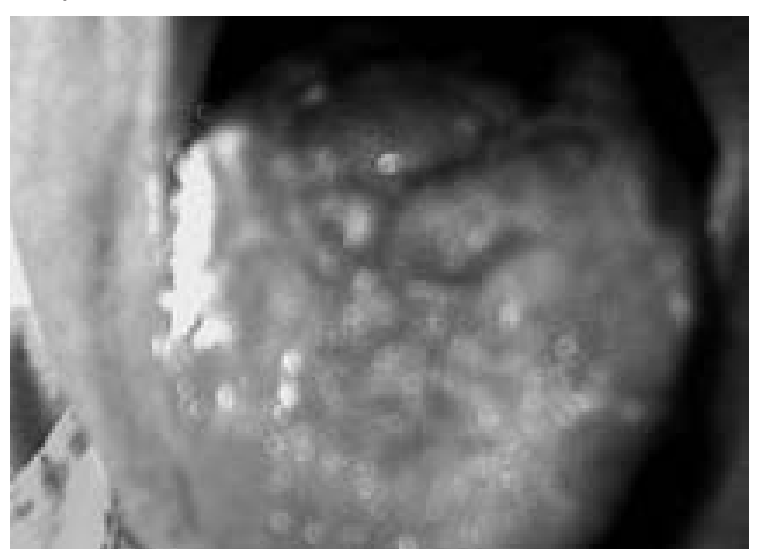

Fig $n^{\circ} 5$ : formation pseudopolypoide de la langue 


\section{DISCUSSION}

La GW est une maladie systémique grave d'origine indéterminée caractérisée par son triple tropisme ORL, pulmonaire et rénal.

Le diagnostic de GW repose sur des arguments cliniques, biologiques et histologiques. La forme typique de la maladie associe classiquement une angéite nécrosante systémique, une inflammation nécrotique des voies aériennes supérieures et inférieures et une glomérulonéphrite extracapsulaire.

L'étiopathogénie de la GW reste discutée, la découverte des auto-anticorps dirigés contre le cytoplasme des polynucléaires neutrophiles (les ANCA) a fait considérer cette maladie comme une véritable maladie auto-immune. La responsabilité d'une atteinte infectieuse comme facteur déclenchant de la maladie ou des rechutes est fortement évoquée $(3,5)$.

Sur le plan histologique, la GW associe trois lésions caractéristiques: une nécrose ischémique, une granulomatose à cellules géantes et une vascularite qui touche les petits et moyens vaisseaux.

L'âge moyen au moment du diagnostic est situé entre 40 à 50 ans avec des extrêmes allant de 9 ans à 78 ans. Cette affection touche presque exclusivement la race blanche sans prédilection de sexe (3).

Les signes inauguraux sont multiples et peuvent être à type de manifestations $O R L$ dans 73 à $83 \%$ des cas, articulaires dans 20 à $60 \%$ des cas et pulmonaires dans 20 à $45 \%$ des cas. Les manifestations oculaires surviennent dans 7 à $41 \%$ des cas et les manifestations cutanées dans 13 à $46 \%$ (3).

Au cours de la maladie de Wegener, l'atteinte oculaire se voit dans 28 à $52 \%$ des cas. Elle est rarement inaugurale et s'associe presque constamment à des lésions des voies aériennes supérieures (6).

Les manifestations oculaires les plus fréquentes sont non spécifiques et les aspects les plus typiques sont par contre rares, telque la pseudotumeur de l'orbite et la sclérite.

Les processus granulomateux orbitaires se manifestent fréquemment par des douleurs, ptosis, œdème palpébral et limitations des mouvements extra-oculaires. Ils sont inauguraux dans uniquement $2 \%$ des cas et observés dans $45 \%$ au cours de l'évolution de la maladie (7).

Comme dans notre première observation, la pseudotumeur de l'orbite est le plus souvent unilatérale.

Son extension serait initialement extracônale avant de devenir intracônale responsable d'une compression du paquet vasculo-nerveux qui peut compromettre la vision. La pseudotumeur inflammatoire est mieux évaluée par le scanner orbitaire ou l'IRM apparaissant en hyposignal en T1 contrastant avec la graisse orbitaire physiologiquement en hypersignal, et également en hyposignal en T2 $(8,9)$.

La biopsie de la lésion orbitaire est parfois nécessaire
Iorsque le tableau clinique et la recherche des ANCA ne permettent pas de poser le diagnostic d'une maladie de Wegener.

Plusieurs diagnostics doivent être évoqués devant toute masse orbitaire. II s'agit de pathologies néoplasiques à type de lymphome orbitaire ou de métastase ; ou de pathologies infectieuses ou inflammatoires (sarcoïdose ou pseudotumeur inflammatoire idiopathique). Les lymphomes et métastases sont généralement iso ou hyposignal en T2 alors qu'en cas de tumeur orbitaire de la GW, elle apparaît en hyposignal.

La localisation laryngée est rare et semble dominer chez les femmes (4).

Elle peut initialiser le tableau dans 7 à $27 \%$ des cas (3). Les lésions siègent habituellement au niveau de l'étage sous glottique réalisant une sténose infiltrante et granulomateuse.

La symptomatologie associe une toux spasmodique et striduleuse, une dyspnée d'effort progressive et parfois une dysphonie.

Le traitement médical est souvent insuffisant, et il faut recourir parfois à des procédés chirurgicaux de désobstruction par endoscopie et laser, calibrage par endoprothèse, laryngoplastie ou encore trachéotomie. Certains auteurs ont proposé l'injection intra-trachéale de corticoïdes avec mise en place d'endoprothèse. Des antibiotiques de type Cotrimoxazole (Pyostacine ${ }^{\circledR}$ ) sont souvent associés pour prévenir les infections et les rechutes (10). Les manifestations endobuccales se retrouvent chez 6 à $13 \%$ des cas $(4,11)$. II peut s'agir d'une gingivite hyperplasique érythémateuse et granuleuse, localisée ou diffuse, ponctuée de taches bleu foncée (gingivite " fraise " (12).

L'évolution aboutit à une atteinte de l'os alvéolaire avec mobilité dentaire.

Les ulcérations se rencontrent habituellement à un stade avancé de la maladie faisant évoquer surtout un cancer de la langue. Une nécrose de la langue est rarement décrite (13).

\section{DISCUSSION}

Toute la particularité de la maladie de Wegener réside dans son évolution capricieuse, son polymorphisme clinique et les difficultés pour affirmer son diagnostic. La place de I'ORL dans le diagnostic est essentielle, il faudra savoir évoquer la maladie devant la persistance de certains signes et devant certaines localisations rares 
LA GRANULOMATOSE DE WEGENER

\section{REFERENCES}

1. Liompart X, Aumaitre O, Kémény J-L, Mom T, Gilain L . Early otorhinolaryngological manifestations of Wegener's granulomatosis :Analysis of 21 patients. Ann Otolaryngol Chir Cervicofac, 2002; 119, 6: 330-336.

2. Cordier J.F. Granulomatose de Wegener. Rev Prat, $2000 ; 50: 271-5$.

3. Costentin B, Dehesdin D, Marie JP, Scarcella-Lecler V, Andrieu-Guitrancourt $\mathrm{J}$. Wegener's granulomatosis involving head and neck: retrospective analysis of 16 patients. Ann Otolaryngol Chir Cervicofac. 2001:118(5):306-14.

4. Metaxaris G, Prokopakis EP, Karatzanis AD, Sakelaris G, Heras P, Velegrakis GA, Helidonis ES. Otolaryngologic manifestations of small vessel vasculitis. Auris Nasus Larynx. 2002;29(4):353-6

5. Boudewyns A, Verbelen J, Koekelkoren E, Van Offel J, Van de Heyning P. Wegener's granulomatosis triggered by infection? J Otolaryngol 2002; 31(6):390-2.

6. Fechner FP, Faquin WC, Pilch BZ. Wegener's granulomatosis of the orbit: a clinicopathological study of 15 patients. Laryngoscope 2002; 112(11):1945-50.

7. Yucel EA, Keles N, Ozturk AS, Solmaz MA, Deger K. Wegener's granulomatosis presenting in the sinus and orbit. Otolaryngol Head Neck Surg 2002; 127(4):349-51.
8. Murphy JM, Gomez-Anson B, Gillard JH, Antoun NM, Cross J, Elliott JD, LockwoodM. Wegener granulomatosis: MR imaging findings in brain and meninges. Radiology 1999; 213(3):794-9.

9. Provenzale JM, Mukherji S, Allen NB, Castillo M, Weber AW. Orbital involvement by Wegener's granulomatosis: imaging findings. Am J Roentgenol 1996; 166(4):929-34.

10. Gluth MB, Shinners PA, Kasperbauer JL. Subglottic stenosis associated with Wegener's granulomatosis. Laryngoscope. 2003;113:1304-7.

11. Chen N, François A, Moguelet P, Péron J-M. Difficult diagnosis for a lingual ulceration.Wegener granulomatosis. Rev Stomatol Chir maxillofac, 2002; 103, 5 : 304-306.

12. Ayache S, Smail A, Stramandinoli E, Pichon J.C , Strunski V. Pharyngeal Wegener's disease: a case report. Ann Otolaryngol Chir Cervicofac,2001;118,3:187-192.

13. Finley JC Jr, Bloom DC, Thiringer JK. Wegener granulomatosis presenting as an infiltrative retropharyngeal mass with syncope and hypoglossal paresis. Arch Otolaryngol Head Neck Surg. 2004;(3):130. 